\title{
Real-Time Liver Motion Compensation for MRgFUS
}

\author{
James C. Ross ${ }^{1}$, Rekha Tranquebar $^{2}$, and Dattesh Shanbhag ${ }^{2}$ \\ ${ }^{1}$ GE Global Research, Niskayuna NY 12309, USA \\ ${ }^{2}$ GE Global Research, Bangalore, Karnataka 560066, IN
}

\begin{abstract}
MR-guided focused ultrasound (MRgFUS) is a non-invasive method by which tissue is ablated using ultrasound energy focused on a point. The procedure has proven effective for stationary targets (e.g. uterine fibroids) but has not yet been used for liver lesion treatment due to organ motion. We describe a method to compensate for organ motion to enable continuous application of ultrasound energy in the presence of target movement in the liver. The method involves tracking several salient features (typically blood vessels) in the vicinity of the target location. The location of the target point(s) themselves are updated using a thin plate spline (TPS) interpolation scheme. We demonstrate sub-pixel tracking accuracy on synthetic sequences and additionally show results on MRI sequences acquired on human subjects. Per-feature tracking times were measured to be $5.7 \mathrm{~ms}$ with a standard deviation of $1.6 \mathrm{~ms}$, sufficient for real-time use.
\end{abstract}

\section{Introduction}

MR-guided Focused Ultrasound (MRgFUS) is a completely non-invasive procedure which uses focused ultrasound energy to ablate lesions deep within the human body. It is typically performed under Magnetic Resonance (MR) imaging guidance. The procedure begins with the acquisition of a planning image which captures the tumor that will be treated. The clinician then manually identifies points on the reference image that indicate where ultrasound energy should be deposited to ablate the tumor. During the procedure, MR images are acquired in order to monitor target temperature changes as well as the temperature of surrounding tissue. MRgFUS has been used to treat stationary targets such as uterine fibroids quite successfully [1], though it has yet to be applied to lesions in moving organs, such as the liver.

Recent efforts have sought to address the issue of organ motion in the context of MRgFUS. In [2], they compute motion fields during a learning phase. Images are acquired before the procedure begins, and motion fields with respect to a reference image are computed offline and stored as an atlas for on-line use. During the procedure, images are continuously acquired, and they are compared to images in the atlas. The motion field corresponding to the most similar image in the atlas is then used. However, MRgFUS procedures can last up to two hours or more. Deformable motion in the liver changes during this time period as a result of muscle relaxation and movements in the bowels. Therefore, relying on precomputed deformation fields derived over a relatively short period of time can prove inaccurate for practical applications.

In [3], they exploit the MRI navigator feature provided by the scanner to determine in-plane translational motion of the moving organ. A Kalman filter tuned to the patientspecific motion characteristics is used to provide accurate and robust results. However,

D. Metaxas et al. (Eds.): MICCAI 2008, Part II, LNCS 5242, pp. 806-813 2008.

(C) Springer-Verlag Berlin Heidelberg 2008 
the navigator only captures translational motion, and although the liver behaves approximately as a rigid-body, non-rigid motion can be non-negligible.

We propose a feature tracking based technique in conjunction with thin plate spline (TPS) interpolation for real-time non-rigid liver motion compensation. Our method uses a tracking algorithm to independently track several salient features (typically blood vessels) in the vicinity of the treatment target. The points defining the treatment target itself are updated using TPS interpolation computed using the tracked feature locations and a corresponding set of reference feature locations. Our paper is layed out as follows: in section 2 we describe our approach and outline a set of experiments used for evaluation. We also give an expression for the TPS error in the context of our algorithm. In section 3 we show experimental results on real and synthetic data. Finally, in section 4 we draw conclusions and discuss future efforts.

\section{Methods}

In addition to the standard workflow used to plan the MRgFUS procedure, we propose an additional step in which salient features (e.g. blood vessels) in the vicinity of the target are also identified. Small regions (a typical size is $15 \times 15$ pixels) centered at each of the manually selected feature locations are then cropped out and stored for subsequent tracking.

Throughout the procedure, real-time image acquisition with MR protocols optimized for feature tracking are collected for motion compensation. It has been shown that liver motion is primarily due to respiration and has dominant motion components in the cranial-caudal and anterior-posterior directions and less motion in the left-right direction [4, 5, 6]. While the liver mostly behaves as a rigid body [7, 8], non-rigid motion is non-negligible [5] and should be handled for accurate target tracking. Therefore, a sagittal acquisition plane that cuts through the treatment region is prescribed for tracking to mitigate out-of-plane motion, and thin plate spline deformation modelling (discussed below) is used for non-rigid motion compensation.

Motion compensation proceeds by independently tracking the feature regions cropped from the planning image throughout the imaging sequence acquired during the procedure. The feature tracking is performed by registering each feature patch to the frame most recently acquired. Registration involves applying a translation transform to the feature patch such that an image-to-image comparison metric is optimized. We have found that a normalized correlation image metric in conjunction with gradient descent optimization produces satisfactory results.

Once the optimal locations of the feature points have been identified within a given frame, thin plate spline interpolation is used to update the locations of the ablation pattern points, defined originally on the reference image. Thin plate splines enable the computation of a continuous deformation field from one image frame (the reference frame) to another frame (acquired during the procedure) given the locations of a sparse set of correspondence points. The correspondence points in this case are the manually defined feature locations on the reference image and the tracked locations of those features in the intra-operatively acquired images. In general it is necessary to provide enough properly located correspondence points to accurately capture the underlying organ deformation. However, given the approximately rigid nature of liver motion, it is sufficient to use only 
a few features. Additionally, while it's possible to compute updated locations for any point in the reference frame using this approach, it's only necessary to compute them for the ablation pattern points. This enables real-time application of the technique and allows the ultrasound focal spot to be steered despite organ motion.

To evaluate the accuracy of the feature tracking algorithm, we tested it on synthetic image sequences as well as MR images of human subjects. The synthetic sequences consisted of a noisy background and a circular foreground object (mimicing a blood vessel) moving in a known sinusoidal manner over several cycles. Additionally, for a given frame in the sequence, the radius was perturbed slightly by a random amount to mimic the pulsatile nature of blood vessels. Figure 1 shows an example synthetic image. We tested radii ranging from 1.0 to 2.0 pixels. For a given radius, we identified the contrast-to-noise (CNR) level that just enabled accurate tracking. Plotting these CNR levels as a function of radius provides a curve below which are

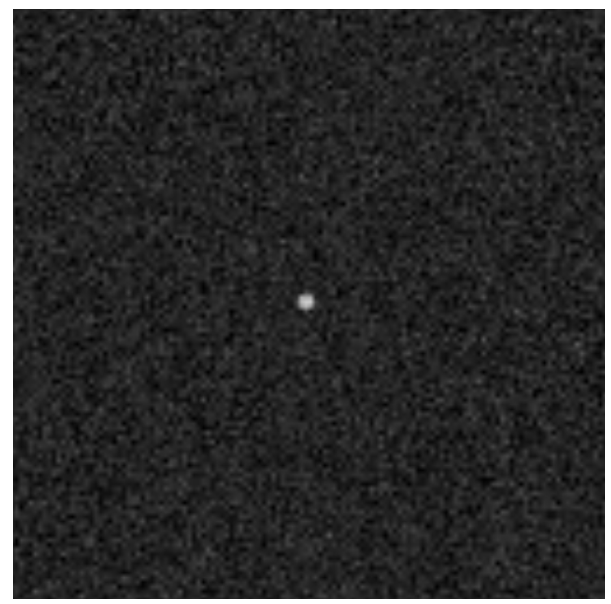

Fig. 1. $128 \times 128$ synthetic image illustrating a vessel-like foreground object with a radius of 1.5 $\mathrm{CNR}$ and radii combinations that break the tracker and above which are combinations that allow for effective tracking. Additionally, at each of the radii / CNR combinations tested along the curve, we computed the mean and standard deviation of the tracking error with respect to the known feature location for each frame.

Once the tracking errors have been established, it is possible to determine the resulting TPS error with respect to a specific point to which the transform is applied. The general expression for the transform can be written as

$$
\mathbf{x}^{\prime}=T\left(\mathbf{x}, \mathbf{p}_{1}, \mathbf{p}_{2}, \cdots, \mathbf{p}_{n}\right)
$$

where $\mathbf{x}$ is the point to transform, $\mathbf{p}_{i}$ are the feature point locations identified in a given frame, and $\mathbf{x}^{\prime}$ is the transformed point. The error in the $i^{t h}$ coordinate of the transformed point is then

$$
\sigma_{x_{i}^{\prime}}=\sqrt{\left(\frac{\partial x_{i}^{\prime}}{\partial p_{11}} \sigma_{p_{11}}\right)^{2}+\cdots+\left(\frac{\partial x_{i}^{\prime}}{\partial p_{n 2}} \sigma_{p_{n 2}}\right)^{2}}
$$

where $p_{i j}$ is the $j^{t h}$ coordinate of the $i^{\text {th }}$ feature location, $\sigma_{p_{i j}}$ corresponds to the error of that coordinate, and $n$ is the number of features being tracked. We assume here that the errors are mutually independent. Since the transform Jacobian is given by

$$
\mathbf{J}=\left[\begin{array}{lllll}
\frac{\partial x_{1}^{\prime}}{\partial p_{11}} & \frac{\partial x_{1}^{\prime}}{\partial p_{1,2}} \cdots \frac{\partial x_{1}^{\prime}}{\partial p_{n 1}} & \frac{\partial x_{1}^{\prime}}{\partial p_{n, 2}} \\
\frac{\partial x_{2}^{\prime}}{\partial p_{11}} & \frac{\partial x_{2}^{\prime}}{\partial p_{12}} \cdots & \cdots & \frac{\partial x_{2}^{\prime}}{\partial p_{n 1}} & \frac{\partial x_{2}^{\prime}}{\partial p_{n 2}}
\end{array}\right]
$$


we can write equation (2) as

$$
\sigma_{x_{j}^{\prime}}=\sqrt{\sum_{i=1}^{2 n} \mathbf{J}_{i j} \mathbf{s} \mathbf{J}_{j i}^{T}}
$$

where $\mathbf{s}=\left[\sigma_{p_{11}} \sigma_{p_{12}} \cdots \sigma_{p_{n 1}} \sigma_{p_{n 2}}\right]^{T}$. A derivation of the TPS Jacobian can be found in [9]. Given typical tracking errors for given radii and CNR combinations, equation (4) provides an expression that will give errors associated with updated ablation pattern points.

\section{Results}

Figure 2 shows CNR as a function of phantom radius. For each CNR-radius pair tested, the mean and standard deviation of the tracking accuracy is shown. Here, tracking accuracy is measured in terms of the magnitude of the error displacement vector. Note that for radii above 1.0 the tracking algorithm was able to perform well. A radius of 1.0 represents the lower limit for this particular experiment, as it was not possible to find a reasonable CNR level that enabled effective tracking over the entire sequence. Note also that the given tracking errors are quite small (sub-pixel accuracy) even though the CNR-radius combinations represented by the curve correspond to values that just enable successful tracking. This suggests that if the algorithm does not lose the feature throughout the sequence, it will track it accurately. Figure 3 shows example plots of

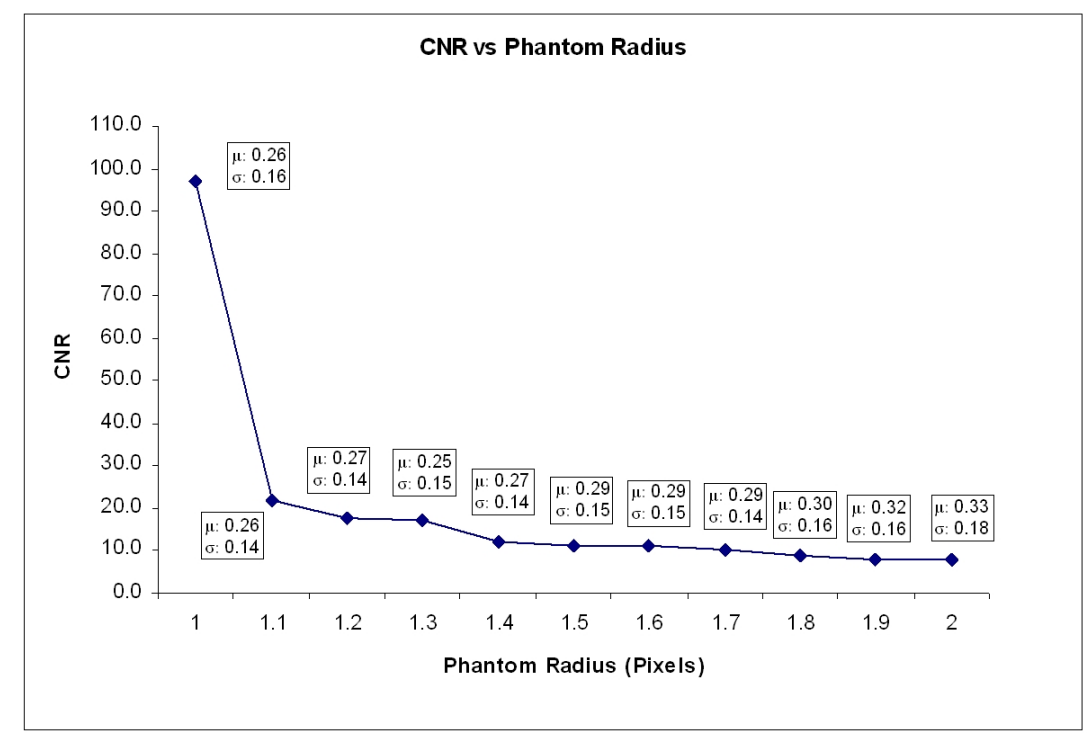

Fig. 2. The curve represents CNR-radius pairs that just enable tracking. For each value pair tested, the corresponding mean and standard deviation of the tracking error is given. 
successful and unsuccessful tracking of the same feature right at the CNR level that enables tracking and just below that level, respectively. Notice that in this particular example, the algorithm is able to reacquire the feature after having lost it. This is not always the case, and for very noisy sequences the track can drift too far away from the correct feature location to reacquire.
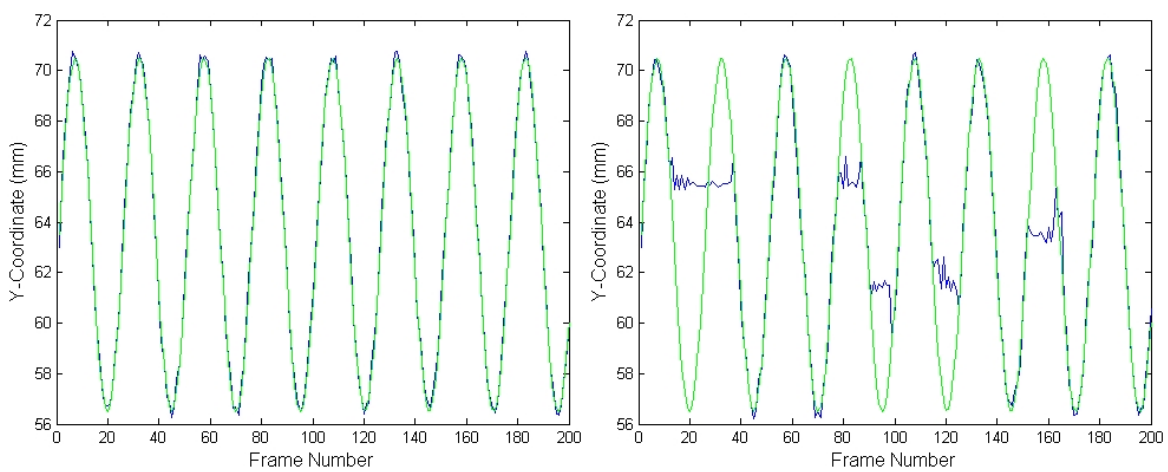

Fig. 3. Example plots of successful (left) and unsuccessful (right) tracking performance of the same feature at slightly different CNR values. The blue plot represents the tracking algorithm output, while the green plot represents ground truth.

In addition to the synthetic sequence studies, we also tested the tracking algorithm on several MRI sequences acquired from human subjects. Each sequence was taken in the sagittal plane, and an MRI protocol that provides blood to liver parenchyma contrast was used. Figure 4 shows results for the sequences tested. The feature regions used for tracking are highlighted with red squares. The center plots show the y-coordinate of each feature location across the entire sequence as determined by the algorithm. The first example illustrates the non-regularity in liver motion that can occur. Such motion can confound techniques that assume periodic liver motion. Though it is not possible to precisely identify the true feature locations in the human sequences, qualitative assesment of the tracking via visual inspection suggests accurate tracking. This conclusion is reinforced by the similarity in shape of the feature coordinate plots. We also used equation (2) to compute the TPS error associated with transforming the centroid of the feature locations in the reference image using the deformation field derived from the reference frame and the frame farthest away from the reference frame. For the vector $\mathrm{s}$ we chose the largest feature localization error computed in the synthetic image sequence study: $\mu=0.33$ and $\sigma=0.18$ to produce an upper bound on the error. TPS errors ranged from 0.5 to $1.5 \mathrm{~mm}$ across the three cases.

In addition to the tracking accuracy studies, we also compiled timing results for the feature tracking algorithm across all three human MRI datasets. Results were generated on a per-feature basis. The mean and standard deviation times to convergence were $5.7 \mathrm{~ms}$ and $1.6 \mathrm{~ms}$, respectively. The mean number of iterations needed to converge was 5.7 with a standard deviation of 1.0. For each new frame in the sequence, the search for each feature location was initiated using the location converged upon in the previous frame. Initializing in this way is sufficient for accurate and fast convergence in most 

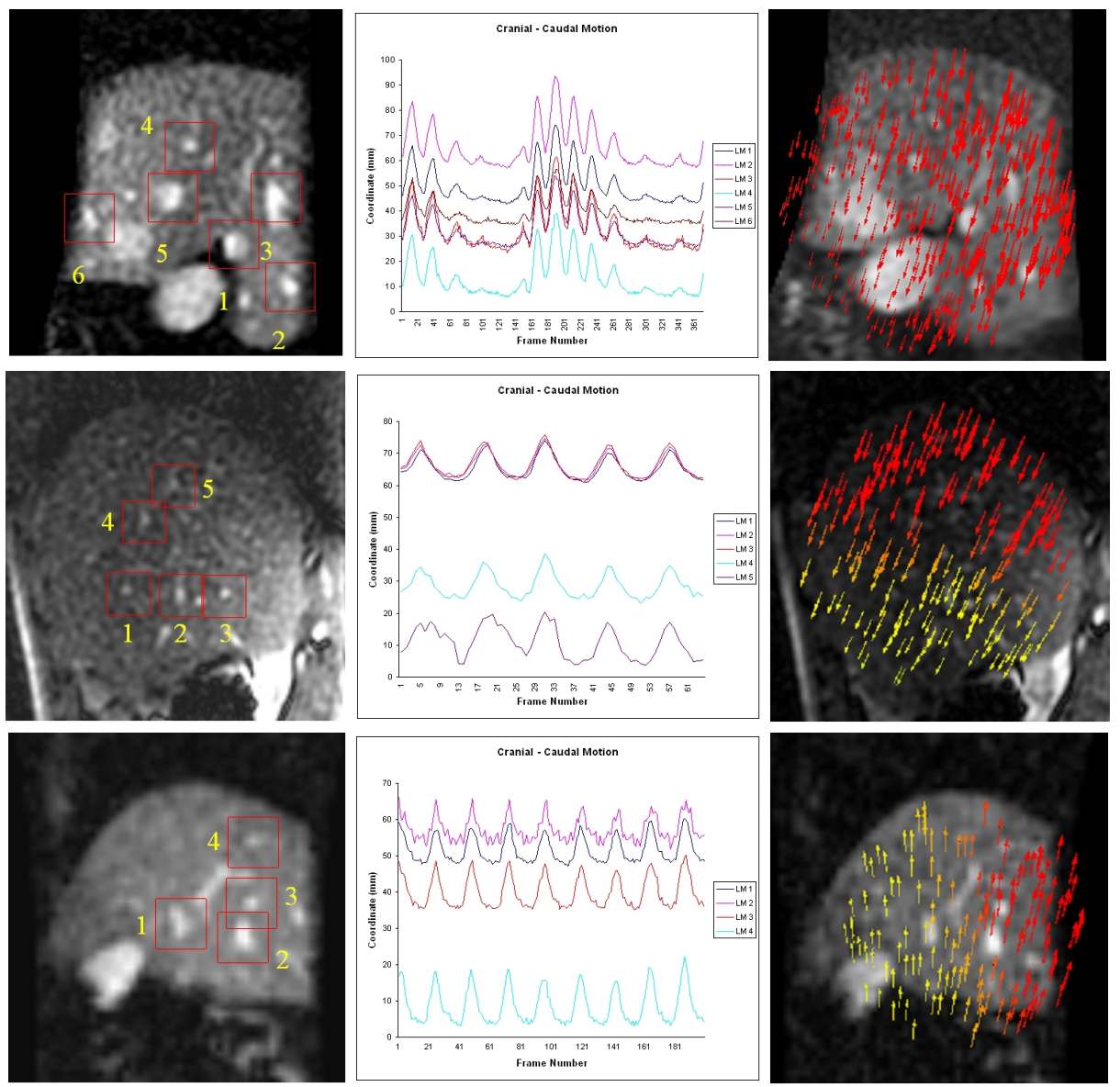

Fig. 4. Left column: sagittal MRI images showing features selected for tracking. Middle column: plots showing the y-coordinate of the tracked feature locations across the entire MRI sequences. Right column: deformation fields computed using TPS showing organ motion from the reference frame to the frame most distant to the reference frame.

cases. However, we should note that tracking performance is sensitive to optimizer settings. We used the Insight Toolkit's [10] implementation of the regular step gradient descent optimizer, which has parameters that control the minimum and maximum step size used during optimization as well as a relaxation factor that controls how quickly the step size shrinks as a minimum is approached (this value ranges from 0.0 to 1.0 with lower values resulting in a more rapid decrease in step-size). We found that using a minimum step size of 0.4 , a maximum step size of 0.7 and a relaxation factor of 0.8 produced good results. These were the values used throughout our experiments to produce the given results. Computation time for ablation pattern position update using TPS is negligible compared to the feature tracking times. This is because TPS need only be applied to the handful of points that define the ablation pattern on the reference image. 


\section{Conclusion}

We have presented a technique for real-time motion compensation that can be applied to MRgFUS for treating liver lesions. The current implementation of our algorithm tracks each feature in series, so the cumulative tracking time is the sum of the times to convergence for each individual feature. However, our approach lends itself well to a multi-threaded implementation, so additional speed-ups can be realized. Next steps include the application of feature coordinate prediction via a Kalman filter, e.g. Accurate feature location prediction will mitigate the issue of latency inherent in a full system integration. We have also not yet explored the use of reliably tracked features to assist with the localization of more noisy features. It stands to reason that the predicted location of a noisy feature can be enhanced with the tracked locations of more reliable features given known, approximate relationships between the features (established, e.g., on the planning image). Reliability of a given feature could potentially be established by the image metric converged upon: the better the metric value, the more reliable the feature's tracked location. Finally, the initialization scheme used in our approach - to start the feature search at the point converged upon in the previous frame - was sufficient for the datasets we tested. However, it's possible that the patient could inhale or exhale rapidly, causing large feature displacement in a short period of time. This could cause our algorithm to lose the track. In [3] the MRI navigator was successfully used to compensate for translational organ motion. Feature tracking initialization could potentially be accomplished using this type of approach. Furthermore, we noted that tracking performance is sensitive to optimizer settings. Tracking could potentially be improved on noisy datasets by dynamically altering optimizer settings so that, e.g., the relaxation factor increased during transitions between inhalation and exhalation, a period that would tend to cause problems due to inadequate initialization. Nevertheless, our algorithm in its current form has shown very accurate and real-time results. We believe this work represents an important contribution to an emerging and exciting procedure.

\section{References}

1. Morita, Y., Ito, N., Hikida, H., Takeuchi, S., Nakamura, K., Ohashi, H.: Non-invasive magnetic resonance imaging-guided focused ultrasound treatment for uterine fibroids - early experience. Eur. J. Obstet. Gynecol. Reprod. Biol. (2007)

2. de Senneville, B., Mougenot, C., Moonen, C.: Real-time adaptive methods for treatment of mobile organs by mri-controlled high-intensity focused ultrasound. Magn. Reson. Med. 57, 319-330 (2007)

3. Lesniak, J., Tokuda, J., Kikinis, R., Burghart, C., Hata, N.: Real-time adaptive methods for treatment of mobile organs by mri-controlled high-intensity focused ultrasound. Phys. Med. Biol. 52, 6427-6438 (2007)

4. Kitamura, K., Shirato, H., Steppenwoolde, Y., Shimizu, T., Kodama, Y., Endo, H., Onimaru, R., Oda, M., Fujita, K., Shimizu, S., Miyasaka, K.: Tumor location, cirrhosis, and surgical history contribute to tumor movement in the liver, as measured during stereotactic irradiation using a real-time tumor tracking radiotherapy system. Int. J. Radiation Oncology Biol. Phys. 56, 221-228 (2003) 
5. Rohlfing, T., Maurer, C., O’Dell, J., Zhong, J.: Modeling liver motion and deformation during the respiratory cycle using intensity-based free-form registration of gated $\mathrm{mr}$ images (2001)

6. Venkatraman, V., Van Horn, M.H., Weeks, S., Bullitt, E.: Liver motion due to needle pressure, cardiac, and respiratory motion during the tips procedure, pp. 66-72 (2004)

7. Clifford, M.A., Banovac, F., Levy, E., Cleary, K.: Assessment of hepatic motion secondary to respiration for computer assisted interventions. Computer Aided Surgery, pp. 291-299 (2002)

8. Sears, W.P.: Development and Application of the New Dynamic NURBS-based CardiacTorso (NCAT) Phantom. PhD thesis, The University of North Carolina (2001)

9. Brooks, R., Arbel, T.: Improvements to the itk:kerneltransform and subclasses. The Insight Journal (2007)

10. Ibanez, L., Schroeder, W., Ng, L., Cates, J.: The ITK Software Guide. Kitware, Inc., 2nd edn. (2005), http: / /www. itk.org/ItkSof twareGuide.pdf ISBN 1-930934-15-7 